Boza Carreño, Ángel; Méndez Garrido, Juan Manuel (2013). Aprendizaje motivado en alumnos universitariōs: validación y resultados generales de una escala. Revista de Investigación Educativa, 31 (2), 331-347.

http://dx.doi.org/10.6018/rie.31.2.163581

\title{
APRENDIZAJE MOTIVADO EN ALUMNOS UNIVERSITARIOS: VALIDACIÓNY RESULTADOS GENERALES DE UNA ESCALA
}

\author{
Ángel Boza Carreño y Juan Manuel Méndez Garrido \\ Universidad de Huelva
}

\section{RESUMEN}

Este trabajo, resultado de un proyecto de investigación financiado por el Plan Propio de Investigación de la Universidad de Huelva, tiene como objetivo construir y validar una escala para evaluar los factores asociados al aprendizaje motivado de los alumnos universitarios. Es una escala Likert que evalúa metas, actitudes, motivos, condiciones, estrategias e implicación en el estudio. La muestra utilizada es aleatoria estratificada proporcional y compuesta por 938 alumnos. Para validar la escala se utiliza juicio de expertos, prueba piloto y análisis factorial. Entre los resultados principales destacamos como rasgos del aprendizaje motivado las metas cognitivas, sociales y de tarea, una actitud orientada al éxito, una atribución de éste a sí mismos, y unos motivos para estudiar relacionados con el trabajo futuro. Los alumnos motivados son competentes en el estudio, combinar capacidad de trabajo individual y trabajo en equipo, usar más las estrategias de aprendizaje, e implicarse en el estudio.

Palabras clave: aprendizaje motivado; motivación académica; metas vitales; actitudes ante el aprendizaje; motivos personales; estrategias de aprendizaje.

\footnotetext{
Correspondencia:

Ángel Boza Carreño. Área de Métodos de Investigación y Diagnóstico en Educación. Departamento de Educación de la Universidad de Huelva. Correo: aboza@uhu.es.

Este trabajo se ha financiado mediante el Plan Propio de Investigación de la UHU-2011.
} 


\section{MOTIVATED LEARNING IN UNIVERSITY STUDENTS: SCALE VALIDATION AND GENERAL RESULTS}

\section{ABSTRACT}

The aim of this study was to develop and validate a scale to assess factors related to college students' motivated learning. The scale was a Likert scale which assessed individuals' goals, attitudes, motives, conditions, strategies and involvement while learning. The sample was proportional stratified random, and comprised 938 students from the University of Huelva, Spain. In the validation process, expert judgment, a pilot test, and factor analysis were used. The most outstanding features of motivated learning were cognitive, social and task goals, a success-oriented attitude, and reasons to study related to future employment. Motivated students excel in their studies, are able to work independently as well as in groups, use learning strategies more often, and get involved in their studies.

Keywords: motivated learning; academic motivation; life goals; attitude to learning; personal reasons; learning strategies.

\section{INTRODUCCIÓN}

Todos necesitamos estar motivados para vivir. La tarea de aprender es una de las constantes vitales del ser humano. Nunca dejamos de aprender por dos razones fundamentales. Necesitamos aprender para poder adaptarnos y modificar nuestro entorno vital. Pero también aprendemos porque queremos aprender, porque queremos conocer los fenómenos que subyacen y explican nuestra vida. No obstante, cuando trasladamos esa necesidad natural a entornos y contenidos formales de educación institucionalizada, no siempre la motivación por aprender cristaliza o se manifiesta con la misma intensidad. Todos conocemos casos de alumnos más o menos motivados o alumnos más motivados en unos momentos que otros. Ello nos hace preguntarnos por la motivación académica de nuestros alumnos universitarios más allá de la dicotomía tradicional de motivación intrínseca y extrínseca.

Pintrich y Schunk (2006) definen la motivación como el proceso que nos dirige hacia el objetivo o la meta de una actividad, que la instiga y la mantiene. Por tanto es más un proceso que un producto, implica la existencia de unas metas, requiere cierta actividad (física o mental), y es una actividad decidida y sostenida.

Habitualmente se ha diferenciado entre motivación extrínseca e intrínseca. La motivación extrínseca es la que lleva a la realización de una tarea como medio para conseguir un fin. Por tanto depende de incentivos externos. Los incentivos extrínsecos proporcionan una satisfacción independiente de la actividad misma. La motivación intrínseca sería la que no depende de incentivos externos, ya que éstos son inherentes a la propia actividad. Las actividades intrínsecamente motivadas son interesantes por sí mismas y no necesitan reforzamiento alguno. No son dos polos contrapuestos y están vinculadas a un momento y contexto (Pintrich y Schunk, 2006). Por último tenemos que señalar que la motivación en educación se ha relacionado siempre con el rendimiento. La motivación también puede influir en el aprendizaje, desarrollando 
una relación recíproca. Si un alumno logra sus metas de aprendizaje, eso le motiva a establecer nuevas metas y desafíos (Meece, 1991). Más recientemente se ha relacionado la motivación con el autoconcepto académico (Isiksal, 2010).

¿Cómo se ha investigado la motivación? Pintrich y Schunk (2006) señalan que los procesos motivacionales se han estudiado desde diferentes metodologías y enfoques: correlacional (Pintrich y De Groot, 1990), experimental (Schunk, 1982), cualitativoetnográfico (Meece, 1991). Por el contrario nosotros hemos encontrado en el entorno español sobre todo estudios cuantitativos basados en cuestionarios y escalas ya probados como el MSQL (Motivated Strategies for Learning Questionnaire-, de Pintrich), usado por Martínez y Galán (2000) y González Cabanach y otros (2007), o el SPQ (Study Process Questionary, de Biggs), utilizado por Hernández y otros (2002). También encontramos estudios basados en otros cuestionarios construidos expresamente para ello (Valle y otros, 1999; Sánchez García, 2001; González Afonso y otros, 2007), casi siempre en formato de escala Likert.

Ya en el entorno académico español, la motivación académica se ha relacionado con diferentes variables centradas en el aprendizaje. En concreto las estrategias de aprendizaje se relacionan con distintas motivaciones (González Gascón y otros, 2010); las metas de logro y las estrategias de autorregulación se relacionan con el rendimiento académico (Salmerón, Gutiérrez Braojos, Salmerón y Rodríguez Fernández, 2011); desde el modelo de enfoques de aprendizaje de Biggs (1987), el motivo profundo correlaciona con estrategias profundas y de alto rendimiento, mientras que el motivo superficial correlaciona con estrategias de alto rendimiento y el motivo de alto rendimiento correlaciona mejor con las estrategias superficiales (Hernández Pina, García, Martínez, Hervás y Maquilón, 2002); González Cabanach, Valle, Rodríguez, García y Mendiri (2007) concluyen que los estudiantes desarrollan metas de aprendizaje para incrementar su capacidad y metas de rendimiento para demostrar su capacidad; Navea (2012) estudió las metas académicas de los alumnos (de tarea, autoensalzamiento, autofrustración y evitación del trabajo) establecidas por Skaalvik en 1997, hallando una correlación positiva entre la meta de tarea y la meta de autoensalzamiento y predominio de la orientación hacia la meta de tarea frente a las demás metas; Sánchez García (2001) señala como motivos de elección de carrera los profesionales antes que los personales; más globalmente Valle, González, Rodríguez, Piñeiro y Suárez (1999) concluyen que los sujetos con niveles de rendimiento alto están más motivados extrínsecamente e intrínsecamente en comparación con los sujetos de rendimiento bajo, atribuyendo su éxito al esfuerzo y a la capacidad.

\section{OBJETIVOS}

- Construir y validar una escala de aprendizaje motivado para universitarios.

- Describir las metas vitales, las actitudes ante el aprendizaje, los motivos personales, las condiciones e implicación en el estudio, y estrategias de aprendizaje de los universitarios. 


\section{METODOLOGÍAY MUESTRA}

Nuestro estudio tiene carácter validativo y descriptivo. Se trata de una encuesta de diseño transversal, vehiculado mediante una escala tipo Likert construida para este estudio. La muestra, compuesta de 938 universitarios de Huelva, extraída mediante muestreo aleatorio estratificado proporcional de las 38 titulaciones de esta universidad, tiene una edad media de 22,17 años (DT=3,93). El 60,1\% son mujeres y el 39,9\% hombres.

\section{INSTRUMENTO A VALIDAR}

Antes de proponer nuestro modelo y diseñar la escala hemos consultados los instrumentos más utilizados en nuestro entorno:

- SPQ (Study Procces Questionnary) de Biggs (1987), utilizado por Hernández, García, Martínez, Hervás y Maquilón (2002). Evalúa motivos y estrategias en tres enfoques de aprendizaje.

- EME-E (Échelle de Motivation en Éducation), de Vallerand, Blais, Brière y Pelletier (1989), validada en español por Núñez, Martín y Navarro (2005).

- MSLQ (Motivational Strategies Learning Questionnaire) de Pintrich, Smith, García y Mckeachie (1991), fundamentado en el modelo de aprendizaje autorregulado y utilizado por Martínez y Galán (2000) y Broc (2011). Traducción y adaptación al español: Cuestionario CEAM II (Roces, Tourón y González, 1995).

- CEVEAPEU (Cuestionario para la evaluación de estrategias de aprendizaje de los estudiantes universitarios), de Gargallo, Suárez-Rodriguez y Pérez-Pérez (2009). Incluye una escala de motivación.

Para nuestro estudio entendemos la motivación académica como motivación consciente para el aprendizaje o como aprendizaje motivado consciente. Nuestro modelo (Figura 1) es ecléctico, fruto tanto de la experiencia docente como de trabajos de investigación previos y en curso. La trama que conforma se refiere a la motivación más personal y consciente del sujeto ante el aprendizaje, aunque no necesariamente intrínseca, e incluye sus actitudes ante el aprendizaje desde las perspectivas de orientación ante el estudio (Atkinson, 1964; Covington y Roberts, 1994), actitud ante la tarea (Wigfield y Eccles, 2000) y atribución de logro, las condiciones en las que desarrolla su estudio, su nivel de implicación en el mismo, las estrategias de aprendizaje que pone en juego (elaboración propia), sus motivos personales (Boza, 2010) y sus metas como ser humano (Ford, 1992). La escala elaborada está compuesta de 84 ítems, distribuidos en 6 dimensiones (figura 1). Algunos resultados generales de este modelo pueden consultarse en Boza y Toscano (2012). 


\begin{tabular}{|c|c|c|}
\hline Dimensiones & \multicolumn{2}{|c|}{ Variables } \\
\hline \multirow{3}{*}{ Metas vitales } & Afectivas & Sociales \\
\hline & Cognitivas & De tarea \\
\hline & Autoasertivas & \\
\hline \multirow{3}{*}{$\begin{array}{l}\text { Actitudes ante el } \\
\text { aprendizaje }\end{array}$} & \multicolumn{2}{|c|}{ Orientación (éxito, fracaso, sobresfuerzo, resignación) } \\
\hline & \multicolumn{2}{|c|}{ Atribución de logro (casualidad, sí mismo, externa) } \\
\hline & \multicolumn{2}{|c|}{ Actitud ante la tarea (expectativa, dificultad, valor) } \\
\hline \multirow{9}{*}{ Motivos personales } & Amistad & Profesión \\
\hline & Aprendizaje & Ayudas \\
\hline & Diversión & Responsabilidad \\
\hline & Experiencia universitaria & Trabajo \\
\hline & Expectativas personales & Bienestar interior \\
\hline & Familia & Superación \\
\hline & Futuro & Importancia del saber \\
\hline & Independencia & Vocación \\
\hline & Mercado de trabajo & Maduración, educación \\
\hline \multirow{3}{*}{$\begin{array}{l}\text { Condiciones del } \\
\text { estudio }\end{array}$} & Competencia & Dedicación \\
\hline & Planificación & Lugar \\
\hline & Individual/grupo & \\
\hline \multirow{5}{*}{$\begin{array}{l}\text { Estrategias de } \\
\text { aprendizaje }\end{array}$} & Conocimientos previos & Síntesis \\
\hline & Fuentes adecuadas & Lecturas personales \\
\hline & Recursos diferentes & Anotaciones \\
\hline & Contenidos relevantes & Materiales complementarios \\
\hline & Lecturas globales & Lecturas diferentes \\
\hline \multirow{4}{*}{$\begin{array}{l}\text { Implicación en el } \\
\text { estudio }\end{array}$} & Esfuerzo & Autonomía \\
\hline & Participación & Asistencia \\
\hline & Resistencia & Persistencia \\
\hline & Responsabilidad & \\
\hline
\end{tabular}

FIGURA 1

MODELO MAM-CEI DE MOTIVACIÓN CONSCIENTE PARA EL APRENDIZAJE

\section{VALIDACIÓN DEL INSTRUMENTO}

La escala ha sido construida en su versión inicial a partir de las referencias consultadas, tras lo cual se ha sometido a un juicio de expertos constituido por alumnos de la licenciatura de Psicopedagogía, alumnos de másteres oficiales y profesores del departamento de educación de la Universidad de Huelva, que evaluaron pertinencia y redacción de los ítems. No se diferenció el papel de alumnos y profesores. A partir de ello se eliminaron algunos ítems por redundancia, mientras que otros se mejoraron en su redacción.

La segunda versión se sometió a análisis de fiabilidad global, entendida como consistencia interna, obteniendo unos valores altos (Alfa de Crombach =0,915). Así mismo se ha analizado cada bloque (tabla 1). Tras su análisis, identificamos los ítems M3, P9 e 
I3 como susceptibles de ser eliminados para su mejora. No obstante su pertinencia en el constructo y su ubicación en dimensiones con un alfa razonable desestiman su eliminación.

TABLA 1

FIABILIDAD DE CADA BLOQUE

\begin{tabular}{|l|c|}
\hline \multicolumn{1}{|c|}{ Bloques } & Alfa de Crombach \\
\hline Metas vitales & 0,855 \\
\hline Actitudes ante el aprendizaje & 0,676 \\
\hline Motivos personales & 0,813 \\
\hline Condiciones del estudio & 0,753 \\
\hline Estrategias de aprendizaje & 0,831 \\
\hline Implicación en el estudio & 0,753 \\
\hline
\end{tabular}

Para el análisis de la validez de constructo hemos realizado un análisis factorial de cada bloque, siguiendo el método de extracción de componentes principales y rotación varimax (no en todos los casos). Previamente se ha calculado el índice KMO (medida de adecuación muestral de Kaiser-Meyer-Olkin) y la prueba de esfericidad de Bartlett, que comprueba que la matriz de correlaciones no es una matriz de identidad. Estos valores resultaron adecuados en todos los casos, haciendo pertinente el análisis factorial. Para determinar el número de factores se ha utilizado el criterio de autovalores superiores a uno. Los resultados han sido los siguientes:

\section{- Metas vitales:}

TABLA 2

METAS VITALES (MATRIZ DE COMPONENTES, ANÁLISIS DE COMPONENTES PRINCIPALES, ROTACIÓN VARIMAX)

\begin{tabular}{|l|c|c|c|c|c|}
\hline $\begin{array}{l}\text { (Varianza total explicada: 61,83\%; KMO=0,749; Prueba de } \\
\text { esfericidad de Barlett: Chi-cuadrado=3189,19; gl=171; Sig.=,000) }\end{array}$ & \multicolumn{5}{|c|}{ Factores } \\
\hline \multicolumn{1}{|c|}{ Ítems } & 1 & 2 & 3 & 4 & 5 \\
\hline M6 &, 743 & & & & \\
M7 &, 762 & & & & \\
M8 &, 771 & & & & \\
M9 &, 643 & & & & \\
\hline M10 &, 475 &, 698 & & & \\
M11 & &, 411 & & & \\
M12 & &, 719 & & & \\
M14 & &, 541 & & & \\
M15 & & &, 455 & &, 492 \\
\hline M19 & & &, 771 & & \\
M20 & & &, 854 & & \\
M21 & & &, 802 & & \\
M22 & & &, 546 & & \\
M13 & &
\end{tabular}




\begin{tabular}{|l|l|l|l|l|l|}
\hline M1 & & & &, 624 & \\
M2 & & & &, 653 & \\
M3 & & & &, 719 & \\
M4 & & & &, 750 & \\
M5 & & & & &, 602 \\
\hline M16 & & & & &, 749 \\
M17 & & & & &, 799 \\
M18 & & & & \\
\hline Porcentaje de varianza explicada por cada factor & 27,10 & 15,56 & 8,82 & 5,57 & 4,77 \\
\hline
\end{tabular}

Se extraen cinco componentes o factores que explican el 61,83\% de la varianza y coinciden con la propuesta inicial, aunque algún ítem cambia de factor:

- Factor 1: Metas cognitivas. M11 (libertad) aparece en este factor, aunque satura también bastante en su bloque inicial (metas autoasertivas).

- Factor 2: Metas autoasertivas.

- Factor 3: Metas de tarea. El ítem M13 (éxito en la vida) pasa a este bloque.

- Factor 4: Metas afectivas.

- Factor 5: Metas sociales. El ítem M19 (competencia), inicialmente meta de tarea, se incluye también aquí, aunque saturando bastante también en el factor 3.

\section{- Actitudes ante el aprendizaje:}

\section{TABLA 3}

ACTITUDES ANTE EL APRENDIZAJE (MATRIZ DE COMPONENTES, ANÁLISIS DE COMPONENTES PRINCIPALES, ROTACIÓN VARIMAX)

\begin{tabular}{|c|c|c|c|c|c|c|}
\hline $\begin{array}{c}\text { (Varianza total explicada: } 57,34 \% ; \text { KMO=,870; Prueba } \\
\text { de esfericidad de Barlett: } \text { Chi-cuadrado=7958,19; gl=231; } \\
\text { Sig.=,000) }\end{array}$ & \multicolumn{6}{|c|}{ Factores } \\
\hline Ítems & 1 & 2 & 3 & 4 & 5 & 6 \\
\hline A5 & ,548 & & & & & \\
\hline A6 & ,577 & & & & & \\
\hline A8 & 777, & & & & & \\
\hline T2 & 765 & & & & & \\
\hline T5 & ,480 & & & & & \\
\hline A1 & & 745 & & & & \\
\hline A2 & & 745 & & & & \\
\hline T6 & & ,536 & & & & \\
\hline A3 & & & 779 & & & \\
\hline A4 & & & 727 & & & \\
\hline A7 & & & ,419 & & & \\
\hline AL1 & & & & 759, & & \\
\hline AL3 & & & & 667 & & \\
\hline AL5 & & & & ,669 & & \\
\hline
\end{tabular}




\begin{tabular}{|l|l|l|l|l|l|l|}
\hline T1 & & & & &, 417 & \\
T3 & & & & &, 799 & \\
T4 & & & & &, 601 & \\
\hline AL2 & & & & & &, 772 \\
AL4 & & & & & &, 827 \\
\hline Porcentaje de varianza explicada por cada factor & 16,84 & 14,36 & 8,29 & 6,66 & 5,78 & 5,40 \\
\hline
\end{tabular}

Se hallan 6 factores que explican el 57,34\% de la varianza y que caracterizamos como actitudes ante el aprendizaje de:

- Factor 1: Resignación ante y expectativa de fracaso (retraso e indiferencia), así como valor de la tarea (interés).

- Factor 2: Orientación al éxito (implicación, confianza), valor de la tarea (dificultad)

- Factor 3: Evitación del fracaso (miedo y ansiedad) y sobreesfuerzo.

- Factor 4: Atribución de logro externa (casualidad, causas externas) y desmotivación.

- Factor 5: Valor de la tarea (coste-facilidad, importancia-utilidad) y capacidad.

- Factor 6: Atribución de logro interna y expectativas positivas.

Estos resultados, aunque concuerdan parcialmente con los factores iniciales (3), nos obligan a revisar la escala para futuras aplicaciones.

\section{- Motivos personales:}

TABLA 4

MOTIVOS PERSONALES (MATRIZ DE COMPONENTES, ANÁLISIS DE COMPONENTES PRINCIPALES)

\begin{tabular}{|l|c|c|c|c|c|}
\hline $\begin{array}{c}\text { (Varianza total explicada: 52,29\%; KMO=,864; } \\
\text { Prueba de esfericidad de Barlett: Chi-cuadrado=4182,24; } \\
\text { gl=190; Sig.=,000) }\end{array}$ & \multicolumn{4}{|c|}{ Factores } \\
\hline \multicolumn{1}{|c|}{ Ítems } & $\mathbf{1}$ & $\mathbf{2}$ & $\mathbf{3}$ & $\mathbf{4}$ & $\mathbf{5}$ \\
\hline P2 &, 574 & & & & \\
P5 &, 612 & & & & \\
P6 &, 424 & & & & \\
P7 &, 520 & & & & \\
P12 &, 615 & & & & \\
P14 &, 661 & & & & \\
P15 &, 723 & & & & \\
P16 &, 685 & & & & \\
P17 &, 562 & & & & \\
P18 &, 635 & & & & \\
P19 &, 448 & & & & \\
P20 &, 454 & & & & \\
\hline
\end{tabular}




\begin{tabular}{|l|l|l|l|l|l|}
\hline P1 &, 444 &, 595 & & & \\
P3 &, 427 &, 663 & & & \\
P4 &, 371 &, 374 & & & \\
\hline P8 &, 353 & &, 357 & & \\
P10 &, 483 & &, 405 & & \\
P13 &, 289 & &, 369 & & \\
\hline P9 &, 283 & & &, 406 & \\
P11 &, 296 & & &, 551 &, 433 \\
\hline Porcentaje de varianza explicada por cada factor & 26,07 & 8,53 & 6,99 & 5,57 & 5,11 \\
\hline
\end{tabular}

Se hallan 5 factores que explican el 52,29\% de la varianza y que caracterizamos como:

- Factor 1: Motivos personales y propios del estudio.

- Factor 2: Motivos de satisfacción en relación a la experiencia universitaria.

- Factor 3: Motivos y exigencias del trabajo/profesión.

- Factor 4: Motivos e incentivos institucionales.

- Factor 5: No se describe el factor porque esos ítems ya saturan en factores anteriores.

Estos resultados coinciden básicamente con la propuesta inicial de un solo factor y nos servirán para repensar la pertinencia de incluir o no en la próxima versión de la escala los ítems de los factores menos personales (P4, P8, P9, P11, P13).

\section{- Condiciones e implicación en el estudio:}

TABLA 5

MOTIVOS PERSONALES (MATRIZ DE COMPONENTES, ANÁLISIS DE COMPONENTES PRINCIPALES)

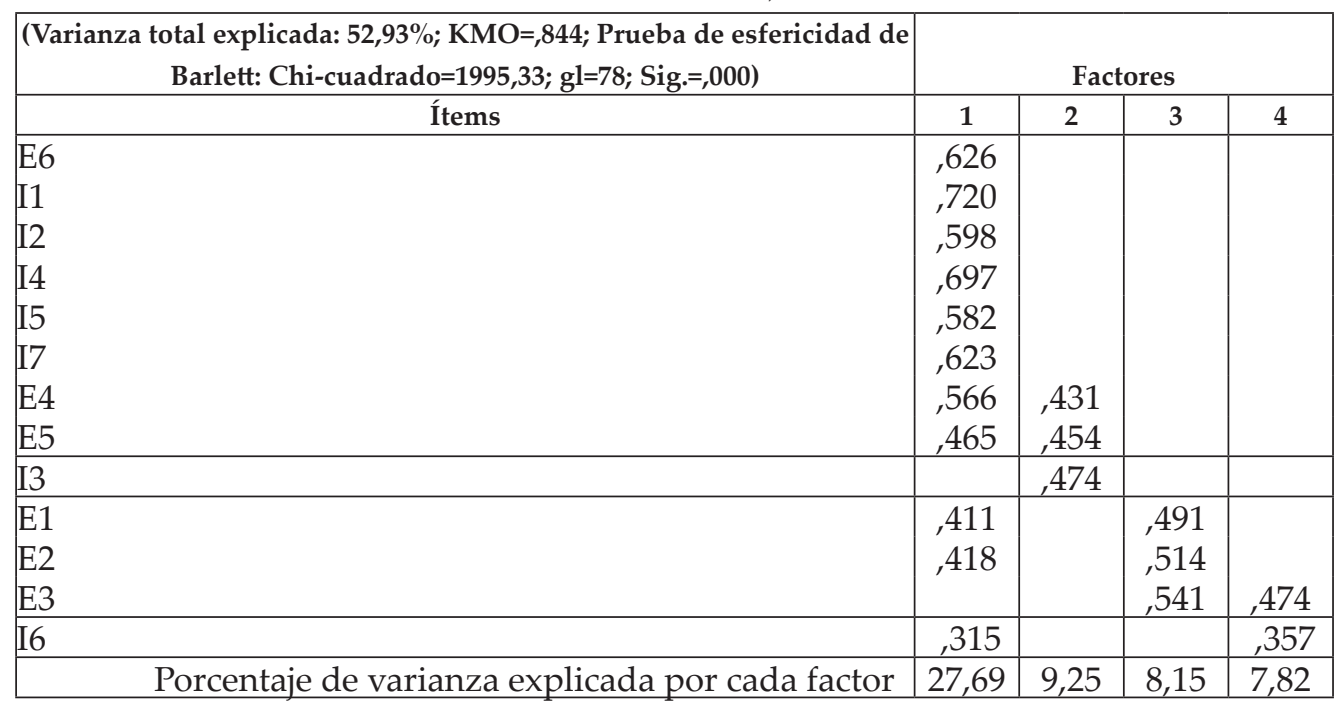


Aunque se hallan 4 factores que explican el 52,93\% de la varianza total, el primero de ellos explica el 27,69\% de la varianza y aglutina a 10 de los 12 ítems del bloque, algunos de los cuales satura también en otro factor. En realidad estaríamos hablando de un solo factor, lo que corrobora el diseño inicial, pero también nos apunta a qué ítems debemos reconsiderar si son pertinentes o no: I3, E3 e I6.

\section{- Estrategias de aprendizaje:}

TABLA 6

MOTIVOS PERSONALES (MATRIZ DE COMPONENTES, ANÁLISIS DE COMPONENTES PRINCIPALES)

\begin{tabular}{|c|c|c|c|}
\hline $\begin{array}{l}\text { (Varianza total explicada: 63,97\%; KMO=,846; } \\
\text { Prueba de esfericidad de Barlett: Chi-cuadrado=2923,04; gl=45; Sig.=,000) }\end{array}$ & \multicolumn{3}{|c|}{ Factores } \\
\hline Ítems & 1 & 2 & 3 \\
\hline EA1 & ,622 & & \\
\hline EA2 & ,675 & & \\
\hline EA3 & ,699 & & \\
\hline EA4 & ,697 & & \\
\hline EA5 & ,717 & & \\
\hline EA6 & ,708 & & \\
\hline EA7 & ,649 & & \\
\hline EA8 &, 567 & & \\
\hline EA9 & ,493 &, 593 & \\
\hline EA10 &, 535 & 415 & ,430 \\
\hline Porcentaje de varianza explicada por cada factor & 41,03 & 12,45 & 10,48 \\
\hline
\end{tabular}

De nuevo nos encontramos con que se hallan 3 factores, que explican el $63,97 \%$ de la varianza total. El primero de ellos explica el $41,03 \%$ de la varianza y aglutina los 10 ítems del bloque, algunos de los cuales satura también en los otros factores. En realidad estaríamos hablando de un solo factor, lo que confirma el diseño inicial, pero también nos apunta a qué ítems podrían ser menos pertinentes: EA9 y EA10.

\section{RESULTADOS GLOBALES}

Examinadas las metas vitales de nuestros alumnos, destacan con medias más altas las metas de tarea (futuro $=5,77$; seguridad $=5,56$; competencia $=5,44$; dinero $=5,42 ;$ éxito $=5,41$ ). Las metas sociales (ayudar $=4,89$; responsabilidad social $=4,79$ ) y cognitivas (saber $=5,17$; comprender $=4,97$ ) ocupan valores intermedios. Niveles bajos presentan las metas afectivas (tranquilidad $=2,98$ ) y algunas metas autoasertivas (competitividad=3,58). Nuestros alumnos estudian para tener un futuro mejor y una vida segura, para ser competentes en su tarea y ganar dinero, para tener éxito en la vida y también para saber. También, pero en menor medida, estudian para poder ayudar a otros, por responsabilidad social, para integrarse en la sociedad y promover la justicia, para comprender el mundo, para desarrollar su creatividad y para mejorar la confianza en sí mismos. 
Dentro de actitudes ante el aprendizaje contemplamos actitudes ante el estudio, actitudes ante la tarea y atribución de logro del mismo. Entre las actitudes ante el estudio, predomina la orientación al éxito $(5,04)$ y el esfuerzo $(4,92)$ sobre la resignación o la evitación del fracaso. Entre las actitudes hacia la tarea, destaca la valoración de la tarea por su utilidad $(5,11)$ frente a su interés, destacando también la expectativa de éxito frente a la expectativa de fracaso. Respecto del logro del aprendizaje, nuestros alumnos se lo atribuyen sobre todo a sí mismos $(5,11)$ frente a la casualidad o a circunstancias externas. En general su rendimiento concuerda con sus expectativas $(4,83)$.

Los motivos más personales de nuestros alumnos son variados: trabajo $(5,79)$, mercado $(5,69)$, expectativas personales (5,54), profesión, el estudio en sí mismo, responsabilidad, conocimiento, superación, vida ordenada, madurez, independencia y singularidad de la experiencia. Curiosamente los valores más bajos, aunque situados en posiciones medias de la escala, corresponden a motivos relacionados con la diversión, los la metodología de los profesores $(4,45)$, la amistad o recibir una beca.

En relación a las condiciones en que realizan su estudio, nuestros universitarios prefieren estudiar solos $(5,02)$ algo más que trabajar en grupo $(4,70)$, se consideran unos estudiantes competentes $(5,01)$ y estudian en lugares apropiados para ello. Las condiciones del estudio menos valoradas son la planificación y el tiempo dedicado al mismo.

Entre las estrategias de aprendizaje más utilizadas por los estudiantes se encuentran la distinción de contenidos relevantes $(5,13)$, las anotaciones $(5,10)$ y el uso de conocimientos previos $(4,97)$. Valores medios alcanzan las estrategias de síntesis, uso de fuentes adecuadas, recursos alternativos y materiales complementarios, así como la realización de lecturas globales y otras lecturas ajenas al contenido del aprendizaje.

Su implicación en el estudio viene determinada por la asistencia a clase $(5,49)$, la responsabilidad, la autonomía y el esfuerzo $(4,97)$. Valores medios alcanzan la participación, la persistencia y la resistencia.

\section{CONCLUSIONES}

Respecto de la validación de la escala, podemos concluir que tenemos un instrumento relativamente válido y fiable. Podemos decir que:

- A pesar de los resultados del análisis de fiabilidad decidimos no eliminar ningún ítem por considerarlos pertinentes en el constructo.

- En la dimensión de metas vitales se confirman cinco factores: cognitivas, autoasertivas, de tarea, afectivas y sociales. El ítem éxito en la vida pasa a meta de tarea y el ítem de competencia pasa a metas sociales.

- En la dimensión actitudes ante el aprendizaje se confirman seis factores, que concuerdan parcialmente con los tres factores iniciales pero combinados: resignación/expectativa de fracaso e interés de la tarea; orientación al éxito y dificultad de la tarea; evitación del fracaso y sobreesfuerzo; atribución de logro externa y desmotivación, valor de la tarea (coste-facilidad, importancia-utilidad) y capacidad; atribución de logro interna y expectativas positivas.

- En la dimensión de motivos personales se hallan cuatro factores: motivos propios, de satisfacción/experiencia universitaria, del trabajo/profesión e incentivos institucionales. Algunos ítems serán eliminados (P4, P8, P9, P11, P13). 
- En la dimensión de condiciones e implicación en el estudio, inicialmente dos, encontramos un solo factor, que aglutina a 10 de los 12 ítems y corrobora el diseño inicial. Se mantienen los ítems por pertinencia en el constructo.

- En la dimensión de estrategias de aprendizaje encontramos un solo factor que confirma el diseño inicial. Los ítems menos pertinentes serían EA9 y EA10.

Globalmente podemos afirmar de nuestros alumnos universitarios:

- Las metas vitales que motivan su estudio se vinculan a la consecución de un futuro mejor, seguridad vital, competencia profesional, éxito económico y personal así como la satisfacción del conocimiento.

- Nuestros alumnos mantienen una actitud ante el estudio orientada al éxito, valorando las tareas de aprendizaje por su importancia y utilidad, y atribuyéndose los logros obtenidos al esfuerzo y trabajo propios.

- Sus motivos personales para estudiar se asocian en el presente con la propia experiencia universitaria como interesante, dar respuesta a sus propias expectativas personales en forma de educación y maduración personal, la adquisición de conocimientos, la propia responsabilidad y los deseos de superación personal, y en el futuro, con la obtención del trabajo deseado, tener mejor opciones en el mercado de trabajo, tener una vida organizada y también disfrutar de independencia y libertad.

- En relación a las condiciones en que realizan su estudio, nuestros universitarios prefieren estudiar solos algo más que trabajar en grupo, se consideran unos estudiantes competentes y estudian en lugares apropiados para ello.

- Entre las estrategias más utilizadas por los estudiantes se encuentran los contenidos relevantes, las anotaciones y el uso de conocimientos previos.

- Su implicación en el estudio viene determinada por la asistencia a clase, la responsabilidad, la autonomía y el esfuerzo.

Estos resultados concuerdan parcialmente con los estudios de Valle (1999), Sánchez García (2001), Hernández Pina (2002), González Cabanach (2007), Navea (2012) y en mayor grado con los de Martínez y Galán (2000), Gargallo (2009, 2010), González Gascón (2010) y Salmerón (2011).

También presentamos en anexo la versión aplicada de la escala, que adoptará en adelante la siguiente estructura: 


\begin{tabular}{|c|c|c|}
\hline Dimensiones & \multicolumn{2}{|c|}{ Variables } \\
\hline \multirow{3}{*}{ Metas vitales } & Afectivas & Sociales \\
\hline & Cognitivas & De tarea \\
\hline & Autoasertivas & \\
\hline \multirow{3}{*}{$\begin{array}{l}\text { Actitudes ante el } \\
\text { aprendizaje }\end{array}$} & Orientación al éxito & Resignación ante fracaso \\
\hline & Valor de la tarea & $\begin{array}{l}\text { Evitación de fracaso y } \\
\text { sobreesfuerzo }\end{array}$ \\
\hline & Atribución de logro interna & Atribución de logro externa \\
\hline \multirow[t]{3}{*}{ Motivos personales } & Personales propios & $\begin{array}{l}\text { Aprendizaje } \\
\text { Expectativas personales } \\
\text { Familia } \\
\text { Independencia } \\
\text { Responsabilidad } \\
\text { Bienestar interior } \\
\text { Superación } \\
\text { Importancia del saber } \\
\text { Vocación } \\
\text { Maduración } \\
\text { Creatividad } \\
\text { Vida organizada } \\
\end{array}$ \\
\hline & $\begin{array}{l}\text { Satisfacción con la } \\
\text { experiencia }\end{array}$ & $\begin{array}{l}\text { Amistad } \\
\text { Diversión }\end{array}$ \\
\hline & Trabajo/Profesión & $\begin{array}{l}\text { Exigencia de estudios } \\
\text { Trabajo deseado }\end{array}$ \\
\hline \multirow{5}{*}{$\begin{array}{l}\text { Condiciones e } \\
\text { Implicación en el } \\
\text { estudio }\end{array}$} & Competencia & Dedicación \\
\hline & Planificación & Lugar \\
\hline & Individuo/Grupo & Responsabilidad \\
\hline & Esfuerzo & Autonomía \\
\hline & Participación & Persistencia \\
\hline \multirow{4}{*}{$\begin{array}{l}\text { Estrategias de } \\
\text { aprendizaje }\end{array}$} & Conocimientos previos & Lecturas globales \\
\hline & Fuentes adecuadas & Síntesis \\
\hline & Recursos diferentes & Lecturas personales \\
\hline & Contenidos relevantes & Anotaciones \\
\hline
\end{tabular}

FIGURA 2

MODELO MAM-CIE DE MOTIVACIÓN CONSCIENTE PARA EL APRENDIZAJE

Para finalizar, creemos que es importante repensar el concepto de aprendizaje motivado o motivación para el aprendizaje, dada su complejidad y multifactorialidad, si queremos modificar los escenarios de aprendizaje de nuestras universidades.

\section{REFERENCIAS}

Alonso, J. (2007). Evaluación de la motivación en entornos educativos. En Bisquerra, R. y Álvarez, M. Manual de Orientación y Tutoría. Wolters Kluwer, Barcelona. 
Atkinson, J.W. (1964). An introduction to motivation. Oxford, England: Van Nostrand.

Boza, A. (2010). Motivación académica en la universidad. En Steren dos Santos, B. y Boza, A. A motivaçao em diferentes cénarios. Porto Alegre, Edipucrs.

Boza, A. y Toscano M.O. (2012). Motivos, actitudes y estrategias de aprendizaje: aprendizaje motivado en alumnos universitarios. Profesorado. Revista de Currículum y Formación del Profesorado. Vol. 16, № 1. http://www.ugr.es/local/recfpro/rev161ART8.pdf

Broc, M.A. (2011). Voluntad para estudiar, regulación del esfuerzo, gestión eficaz del tiempo y rendimiento académico en alumnos universitarios. Revista de Investigación Educativa, 29(1), 171-185.

Closas, A.; Sanz, M.L. y Ugarte, M.D. (2011). An Explanatory Model of the Relations Between Cognitive and Motivational Variables and Academic Goals. Revista de Psicodidáctica, 2011, 16(1), 19-38 ISSN 1136-1034. http://www.ehu.es/revista-psicodidactica

Covington, M.V.; Roberts, B. W. (1994). Self-worth and college achievement: Motivational and personality correlates. En Pintrich, Paul R. (Ed); Brown, Donald R. (Ed); Weinstein, Claire Ellen (Ed), (1994). Student motivation, cognition, and learning: Essays in honor of Wilbert J. McKeachie, (pp. 157-187). Hillsdale, NJ, England: Lawrence Erlbaum Associates.

Ford, M.E. (1992). Motivating humans: goals, emotions, and personal agency beliefs. Newbury Park, SAGE Publications.

Gargallo, Bernardo, Suárez-Rodríguez, Jesús M. y Pérez-Pérez, Cruz (2009). E1 cuestionario CEVEAPEU. Un instrumento para la evaluación de las estrategias de aprendizaje de los estudiantes universitarios. RELIEVE, v. 15, n. 2, p. 1-31. http://www.uv.es/RELIEVE/v15n2/RELIEVEv15n2_5.htm

Gargallo, B.; Garfella, P.; Pérez Pérez, C.; Fernández March, A. (2010). Modelos de enseñanza y aprendizaje en la universidad. (Ponencia III. Seminario Interuniversitario de Teoría de la Educación, Madrid 2010). http://www.uv.es/gargallo/Modelos2.pdf

González Afonso, M.C., Álvarez, P.R., Cabrera, L. y Bethencourt, J.T. (2007). El abandono de los estudios universitarios: factores determinantes y medidas preventivas. Revista Española de Pedagogía, nº 236, 49-70. http://revistadepedagogia.org/2007060131/ vol.-lxv-2007/n\%C2\%BA-236-enero-abril-2007/el-abandono-de-los-estudios-universitarios-factores-determinantes-y-medidas-preventivas.html

González Cabanach, R., Valle, A., Rodríguez, S., García, M. y Mendiri, P. (2007). Programa de intervención para mejorar la gestión de recursos motivacionales en estudiantes universitarios. Revista Española de Pedagogía, $n^{-}$237, 237-256. http://revistadepedagogia.org/20071003328/vol.-1xv-2007/n\%C2\%BA-237-mayo-agosto-2007/ programa-de-intervencion-para-mejorar-la-gestion-de-los-recursos-motivacionalesen-estudiantes-universitarios.html

González Fernández, A. (2007). Modelos de motivación académica: una visión panorámica. Revista Electrónica de Motivación y Emoción. Vol X, nํ 25. http://reme.uji.es/ articulos/numero25/article1/article1.pdf

González Gascón, E.; De Juan, M.D.; Parra, J.F.; Sarabia, F.J. y Kanther, A. (2010). Aprendizaje autorregulado: antecedentes y aplicación a la docencia universitaria de marketing. Revista de Investigación Educativa, 28(1), 171-194. http://revistas.um.es/ rie/article/view/97831.

Hernández, F., García, M.P., Martínez, P., Hervás, R.M. y Maquilón, J. (2002). Consistencia entre motivos y estrategias de aprendizaje en estudiantes universitarios. 
Revista de Investigación Educativa, v.20, 487-510. http://www.doredin.mec.es/documentos/007200330333.pdf.

Isiksal, M. (2010). A comparative Study on Undergratuate Students' Academic Motivation and Academic Self-Concept. The Spanish Journal of Psychology, Vol. 13, № 2, 572-585. http://www.ncbi.nlm.nih.gov/pubmed/20977008.

Martínez, J.R. y Galán, F. (2000). Estrategias de aprendizaje, motivación y rendimiento académico en alumnos universitarios. Revista de Española de Orientación y Psicopedagogía, vol. 11, no 19, 35-45. http://www.uned.es/reop/pdfs/2000/11-19-1---035-Reynaldo\%20Martinez.PDF.

Meece, J. (1991). The classroom context and students' motivational goals. In Maehr, M.L. y Pintrich, P.R. (Eds). Advances in motivation and achievement, vol. 7, 261-286.

Navea, A. (2012). Un Estudio sobre las Metas Académicas en Estudiantes Universitarios de Enfermería. Psicología Educativa. Vol. 18, n. ${ }^{\circ}$ 1, 2012 - Págs. 83-89. http://www. copmadrid.org/webcopm/publicaciones/educativa/ed2012v18n1a8.pdf.

Núñez, J.L.; Martín, J. y Navarro, J.G. (2005). Validación de la versión española de Échelle de motivation en éducation. Psicothema, vol. 17, no 002, 344-349.

Pintrich, P.R. y De Groot, E. (1990). Motivational and self-regulated learning components of classroom academia performance. Journal of Educational Psychology, 82, 33-40.

Pintrich, P.R. y Schunk, D.H. (2006). Motivación en contextos educativos. Madrid, Pearson.

Salmerón, H.; Gutiérrez Braojos, C.; Salmerón, P. y Rodríguez Fernández, S. (2011). Metas de logro, estrategias de regulación y rendimiento académico en diferentes estudios universitarios. Revista de Investigación Educativa, 29(2), 467-486. http:// revistas.um.es/rie/article/view/116041.

Sánchez García, M.F. (2001). La orientación universitaria y las circunstancias de elección de los estudios. Revista de Investigación Educativa, v. 19, nª 1, 39-61. http://www. doredin.mec.es/documentos/007200230123.pdf.

Schunk (1982). Effects of effort atributional feedback on children's perceived self-efficacy and achievement. Journal of Educational Psychology, 74, 548-556.

Suárez, J.M. y Fernández Suárez, A.P. (2005). Escalas de evaluación de las estrategias motivacionales de los estudiantes. Anales de Psicología, vol. 21, no 1, 116-128. http:// digitum.um.es/jspui/handle/10201/8054

Torrano, F. y González Torres, M.C. (2004). El aprendizaje autorregulado: presente y futuro de la investigación. Revista Electrónica de Investigación Psicoeducativa, 2(1), 1-34. http:// www.investigacion-psicopedagogica.org/revista/articulos/3/espannol/Art_3_27.pdf

Valle, A., González, R., Rodríguez, S., Piñeiro, I. y Suárez, J.M. (1999). Atribuciones causales, autoconcepto y motivación en estudiantes con alto y bajo rendimiento académico. Revista Española de Pedagogía, nº 214, 525-546. http://revistadepedagogia.org/20070607223/ vol.-lvii-1999/n\%C2\%BA-214-septiembre-diciembre-1999/atribuciones-causales-autoconcepto-y-motivacion-en-estudiantes-con-alto-y-bajo-rendimiento-academico.html

Wigfield, A. y Eccles, J.S. (2000). Expectancy-Value Theory of Achievement Motivation. Contemporary Educational Psychology, Volume 25, Issue 1, January 2000, Pages 68-81. http://www.sciencedirect.com/science/article/pii/S0361476X99910159.

Fecha de recepción: 27 de noviembre de 2012.

Fecha de revisión: 29 de noviembre de 2012.

Fecha de aceptación: 7 de marzo de 2013. 


\section{ANEXO:}

\section{ESCALA DEAUTOPERCEPCIÓN DE LA MOTIVACIÓN ACADÉMICA PERSONAL}

A continuación encontrarás una serie de afirmaciones sobre tu vida como alumno. Te pedimos que nos señales tu grado de acuerdo o desacuerdo con cada una de ellas, sabiendo que: $1=$ Nada de acuerdo; 7=Totalmente de acuerdo.

\begin{tabular}{|c|c|c|c|c|c|c|c|c|}
\hline \multirow{22}{*}{$\underbrace{}_{\substack{\frac{\pi}{0} \\
\Sigma}}$} & M1. Estudio para sentirme activo, para evitar aburrirme & 1 & 2 & 3 & 4 & 5 & 6 & 7 \\
\hline & M2. Estudio para tranquilizarme, para evitar el stress & 1 & 2 & 3 & 4 & 5 & 6 & 7 \\
\hline & M3. Estudio porque me hace ser más feliz & 1 & 2 & 3 & 4 & 5 & 6 & 7 \\
\hline & M4. Estudiar me produce satisfacción & 1 & 2 & 3 & 4 & 5 & 6 & 7 \\
\hline & M5. Estudiar me hace sentirme sano, fuerte, enérgico & 1 & 2 & 3 & 4 & 5 & 6 & 7 \\
\hline & M6. Estudio para saber & 1 & 2 & 3 & 4 & 5 & 6 & 7 \\
\hline & M7. Estudio para comprender en profundidad la materia & 1 & 2 & 3 & 4 & 5 & 6 & 7 \\
\hline & M8. Estudio para desarrollar mi creatividad intelectual & 1 & 2 & 3 & 4 & 5 & 6 & 7 \\
\hline & M9. Estudio para mejorar la confianza en mí mismo & 1 & 2 & 3 & 4 & 5 & 6 & 7 \\
\hline & M10. Estudio para sentirme único, diferente a los demás & 1 & 2 & 3 & 4 & 5 & 6 & 7 \\
\hline & M11. Estudio para ser más libre en mis opiniones y decisiones & 1 & 2 & 3 & 4 & 5 & 6 & 7 \\
\hline & M12. Estudio para ser mejor que los demás & 1 & 2 & 3 & 4 & 5 & 6 & 7 \\
\hline & M13. Estudio para tener éxito en la vida & 1 & 2 & 3 & 4 & 5 & 6 & 7 \\
\hline & M14. Estudio para ser valorado por los demás & 1 & 2 & 3 & 4 & 5 & 6 & 7 \\
\hline & M15. Estudio para integrarme en la sociedad & 1 & 2 & 3 & 4 & 5 & 6 & 7 \\
\hline & M16. Estudio para asumir una responsabilidad social & 1 & 2 & 3 & 4 & 5 & 6 & 7 \\
\hline & M17. Estudio para promover la justicia y la equidad & 1 & 2 & 3 & 4 & 5 & 6 & 7 \\
\hline & M18. Estudio para poder ayudar a otros & 1 & 2 & 3 & 4 & 5 & 6 & 7 \\
\hline & M19. Estudio para ser competente en mi materia o tarea & 1 & 2 & 3 & 4 & 5 & 6 & 7 \\
\hline & M20. Estudio para tener un futuro mejor & 1 & 2 & 3 & 4 & 5 & 6 & 7 \\
\hline & M21. Estudio para ganar dinero & 1 & 2 & 3 & 4 & 5 & 6 & 7 \\
\hline & M22. Estudio para tener una vida segura & 1 & 2 & 3 & 4 & 5 & 6 & 7 \\
\hline \multirow{18}{*}{ 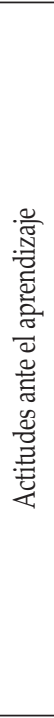 } & A1. Me implico mucho en las actividades académicas & 1 & 2 & 3 & 4 & 5 & 6 & 7 \\
\hline & A2. Confío en obtener buenas calificaciones & 1 & 2 & 3 & 4 & 5 & 6 & 7 \\
\hline & A3. Tengo miedo a fracasar en mis estudios & 1 & 2 & 3 & 4 & 5 & 6 & 7 \\
\hline & A4. Estudiar me genera ansiedad & 1 & 2 & 3 & 4 & 5 & 6 & 7 \\
\hline & A5. Acostumbro a retrasarme en la realización de las tareas académicas & 1 & 2 & 3 & 4 & 5 & 6 & 7 \\
\hline & A7. Me preocupa hacer todo bien & 1 & 2 & 3 & 4 & 5 & 6 & 7 \\
\hline & A8. Me es indiferente conseguir realizar bien la tarea & 1 & 2 & 3 & 4 & 5 & 6 & 7 \\
\hline & T1. Las tareas fáciles me parecen más atractivas & 1 & 2 & 3 & 4 & 5 & 6 & 7 \\
\hline & T2. Hago solamente las tareas que me interesan & 1 & 2 & 3 & 4 & 5 & 6 & 7 \\
\hline & T3. Me esfuerzo en las tareas que considero importantes o útiles & 1 & 2 & 3 & 4 & 5 & 6 & 7 \\
\hline & T4. Siempre pienso que soy capaz de hacer bien la tarea & 1 & 2 & 3 & 4 & 5 & 6 & 7 \\
\hline & T5. Las tareas desbordan habitualmente mi capacidad & 1 & 2 & 3 & 4 & 5 & 6 & 7 \\
\hline & T6. Las dificultades me motivan, suponen un reto para mí & 1 & 2 & 3 & 4 & 5 & 6 & 7 \\
\hline & AL1. Atribuyo mis éxitos o fracasos a la casualidad & 1 & 2 & 3 & 4 & 5 & 6 & 7 \\
\hline & AL2. Atribuyo mis éxitos a mí mismo & 1 & 2 & 3 & 4 & 5 & 6 & 7 \\
\hline & AL3. Atribuyo mis fracaso a consecuencias externas a mí & 1 & 2 & 3 & 4 & 5 & 6 & 7 \\
\hline & AL4. Mi rendimiento se asemeja a mis expectativas & 1 & 2 & 3 & 4 & 5 & 6 & 7 \\
\hline & AL5. Me desmotivo fácilmente & 1 & 2 & 3 & 4 & 5 & 6 & 7 \\
\hline
\end{tabular}




\begin{tabular}{|c|c|c|c|c|c|c|c|c|}
\hline \multirow{20}{*}{ 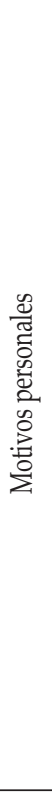 } & P1. Estudio para conocer gente nueva y entablar amistades & 1 & 2 & 3 & 4 & 5 & 6 & 7 \\
\hline & P2. Estudio para aprender y avanzar en mis conocimientos & 1 & 2 & 3 & 4 & 5 & 6 & 7 \\
\hline & P3. Estudio en la universidad porque me divierto también mucho & 1 & 2 & 3 & 4 & 5 & 6 & 7 \\
\hline & P4. La vida del estudiante es una experiencia única y muy gratificante & 1 & 2 & 3 & 4 & 5 & 6 & 7 \\
\hline & P5. Estudio porque quiero que se cumplan mis expectativas & 1 & 2 & 3 & 4 & 5 & 6 & 7 \\
\hline & P6. La influencia y apoyo de mi familia hizo que me decidiera a estudiar & 1 & 2 & 3 & 4 & 5 & 6 & 7 \\
\hline & P7. Estudio porque así puedo vivir con más independencia y libertad & 1 & 2 & 3 & 4 & 5 & 6 & 7 \\
\hline & P8. Estudio porque va a aumentar mis posibilidades en el mercado de trabajo & 1 & 2 & 3 & 4 & 5 & 6 & 7 \\
\hline & P9. Mis profesores y su metodología me motivan para seguir estudiando. & 1 & 2 & 3 & 4 & 5 & 6 & 7 \\
\hline & P10. Estudio porque la profesión que quiero ejercer requiere estos estudios & 1 & 2 & 3 & 4 & 5 & 6 & 7 \\
\hline & P11. Recibir una beca es un motivo para seguir estudiando & 1 & 2 & 3 & 4 & 5 & 6 & 7 \\
\hline & P12. Mis estudios me hacen sentir más responsable & 1 & 2 & 3 & 4 & 5 & 6 & 7 \\
\hline & P13. Decidí estudiar esta carrera para trabajar en algo que me gusta & 1 & 2 & 3 & 4 & 5 & 6 & 7 \\
\hline & P14. Estudio porque me hace sentirme bien conmigo mismo/a & 1 & 2 & 3 & 4 & 5 & 6 & 7 \\
\hline & P15. Me motiva comprobar que soy capaz de superarme a mí mismo & 1 & 2 & 3 & 4 & 5 & 6 & 7 \\
\hline & P16. Pienso que los estudios son muy importante, por eso estudio & 1 & 2 & 3 & 4 & 5 & 6 & 7 \\
\hline & P17. Estudio por vocación, porque siempre quise estudiar esta carrera & 1 & 2 & 3 & 4 & 5 & 6 & 7 \\
\hline & P18. Estudio para educarme, para madurar como persona & 1 & 2 & 3 & 4 & 5 & 6 & 7 \\
\hline & P19. Me gusta implicarme en actividades creativas & 1 & 2 & 3 & 4 & 5 & 6 & 7 \\
\hline & P20. Prefiero llevar una vida ordenada, organizada & 1 & 2 & 3 & 4 & 5 & 6 & 7 \\
\hline \multirow{12}{*}{ 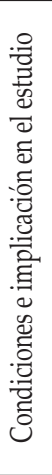 } & E1. Me considero un estudiante competente & 1 & 2 & 3 & 4 & 5 & 6 & 7 \\
\hline & E2. Prefiero estudiar solo & 1 & 2 & 3 & 4 & 5 & 6 & 7 \\
\hline & E3. El trabajo de grupo me parece eficaz & 1 & 2 & 3 & 4 & 5 & 6 & 7 \\
\hline & E4. Elaboro un horario previo de estudio y actividades & 1 & 2 & 3 & 4 & 5 & 6 & 7 \\
\hline & E5. Suelo dedicarle bastante tiempo al estudio (4h/día o más) & 1 & 2 & 3 & 4 & 5 & 6 & 7 \\
\hline & E6. Estudio en un lugar adecuado para ello & 1 & 2 & 3 & 4 & 5 & 6 & 7 \\
\hline & I1. Me esfuerzo en mis estudios todo lo que puedo & 1 & 2 & 3 & 4 & 5 & 6 & 7 \\
\hline & I2. Participo habitualmente en las clases & 1 & 2 & 3 & 4 & 5 & 6 & 7 \\
\hline & I4. Me considero responsable como estudiante & 1 & 2 & 3 & 4 & 5 & 6 & 7 \\
\hline & I5. Como estudiante soy bastante autónomo & 1 & 2 & 3 & 4 & 5 & 6 & 7 \\
\hline & I6. Asisto a clase todos los días & 1 & 2 & 3 & 4 & 5 & 6 & 7 \\
\hline & I7. Suelo llevar las asignaturas al día & 1 & 2 & 3 & 4 & 5 & 6 & 7 \\
\hline \multirow{10}{*}{ 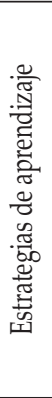 } & EA1. Utilizo mis conocimientos previos & 1 & 2 & 3 & 4 & 5 & 6 & 7 \\
\hline & EA2. Selecciono las fuentes adecuadas & 1 & 2 & 3 & 4 & 5 & 6 & 7 \\
\hline & EA3. Utilizo recursos diferentes & 1 & 2 & 3 & 4 & 5 & 6 & 7 \\
\hline & EA4. Soy capaz de distinguir los contenidos más relevantes & 1 & 2 & 3 & 4 & 5 & 6 & 7 \\
\hline & EA5. Realizo lecturas globales & 1 & 2 & 3 & 4 & 5 & 6 & 7 \\
\hline & EA6. Realizo síntesis de los contenidos & 1 & 2 & 3 & 4 & 5 & 6 & 7 \\
\hline & EA7. Hago lecturas personales & 1 & 2 & 3 & 4 & 5 & 6 & 7 \\
\hline & EA8. Realizo anotaciones al margen (o en un papel) cuando leo o estudio & 1 & 2 & 3 & 4 & 5 & 6 & 7 \\
\hline & EA9. Destino parte de mi tiempo libre a otras lecturas & 1 & 2 & 3 & 4 & 5 & 6 & 7 \\
\hline & EA10. Utilizo materiales complementarios & 1 & 2 & 3 & 4 & 5 & 6 & 7 \\
\hline
\end{tabular}

¡Gracias por tu colaboración y sinceridad! 
